\title{
BMJ Open Quality Using the plan-do-study-act approach to improve inpatient colonoscopy preparation
}

\author{
Sardar Musa Shah-Khan, ${ }^{1}$ Jeremy Cumberledge ${ }^{2}$ Gorman Joel Reynolds ${ }^{2}$
}

To cite: Shah-Khan SM, Cumberledge J, Reynolds GJ. Using the plan-do-studyact approach to improve inpatient colonoscopy preparation.BMJ Open Quality 2017;6:e000230. doi:10.1136/ bmjoq-2017-000230

Received 14 October 2017 Revised 21 November 2017 Accepted 21 November 2017
CrossMark

${ }^{1}$ Department of Medicine, West Virginia University, Morgantown, West Virginia, USA

${ }^{2}$ Department of Digestive Diseases, West Virginia University, Morgantown, West Virginia, USA

Correspondence to Dr Sardar Musa Shah-Khan; mshahkhan@gmail.com

\section{ABSTRACT}

Poor inpatient colonoscopy preparations can provide multiple challenges to healthcare providers and patients alike. Poor preparations can make the colonoscopy difficult to perform, and can require the procedure to be repeated. This can in turn lead to greater costs, longer length of stays, less patient satisfaction and worse outcomes. The aim of this quality improvement project was to decrease the rate of poor inpatient colonoscopy preparations using the plan-do-study-act approach. Inpatient colonoscopies at our institution from a 3-month span (November 2016 to January 2017) were evaluated, and found to have a $19 \%$ rate of poor preparations. A multiphase intervention programme was then conducted to improve the quality of these preparations. This intervention programme was threefold, and involved (1) direct education to physicians and nursing staff on the preparation process and its importance; (2) the implementation of an electronic order set within our electronic medical record (EMR) to standardise and simplify the process of ordering colonoscopy preparations; and (3) patient education in the form of a handout explaining the steps and importance of a good preparation. Through these interventions, we were able to bring down our rate of poor preparations over a 3-month average from $19 \%$ to $4 \%$. Specifically, the implementation of an electronic order set within our EMR resulted in the greatest impact. Our interventions can be replicated at other institutions in order to decrease the rate of poor preparations, and thus result in better outcomes for patients, providers and healthcare facilities.

\section{PROBLEM}

When looking at possible quality improvement projects within the Department of Digestive Diseases at West Virginia University, we realised that there were ways to improve the quality of one of the most common procedures performed at our institution: colonoscopies. Anecdotally, we noticed that many times, the colonoscopy preparations at our institution that were completed were poor, and thus not adequate enough to complete the procedure. This in turn led to delayed procedures, repeat preparations and longer patient stays. At a time when healthcare resources and spending are at a premium, finding a way to reduce these poor preparations became a goal. The purpose of this project was thus to decrease the rate of poor inpatient colonoscopy preparations at our institution starting in February 2017 until July 2017.

J.W. Ruby Memorial Hospital is the flagship hospital of the West Virginia University Health System. It is a 645-bed level 1 trauma and academic medical centre in Morgantown, WV. The WVU Department of Digestive Diseases is responsible for conducting the vast majority of inpatient colonoscopies at Ruby Memorial, and thus it was this department that undertook this quality improvement project. The project was conducted by three individuals: a resident in the Department of Internal Medicine, and a fellow and attending within the Department of Digestive Diseases.

\section{BACKGROUND}

Colonoscopies are a critical part of screening for colon cancer, as well as looking for cause of lower gastrointestinal (GI) bleeding in patients. A successful colonoscopy requires an adequate preparation. There are various preparations available to patients in the inpatient setting. These include GoLytely (polyethylene glycol and electrolytes for oral solution) and Miralax (polyethylene glycol). Administering these preparations to patients has traditionally been difficult, as these preparations are known for having a poor taste and require large amounts to be successful.

The quality of colonoscopy preparations is an important factor in determining whether or not the procedure can be performed. One study showed that $11 \%$ of incomplete colonoscopies were due to poor preparation. ${ }^{1}$ A poor preparation can be defined as one in which the bowel cannot be adequately visualised, and/or requires a lot of cleaning. Poor preparations can subsequently lead to repeat procedures, which can increase patient length of stay and healthcare costs. Thus, ensuring an adequate colonoscopy preparation is essential in determining whether the procedure 


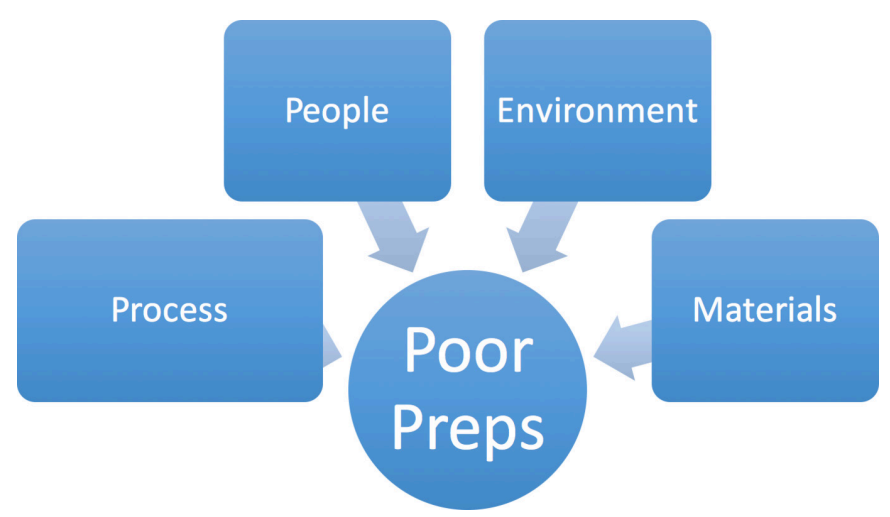

Figure 1 Factors contributing to poor colonoscopy preparations at an inpatient institution.

can be completed or not. The plan-do-study-act (PDSA) approach has been used at other facilities with success for outpatient colonoscopy preparations. ${ }^{2}$ Our project sought to intervene on colonoscopy preparations in the inpatient setting, as this environment can provide more complexity in terms of the factors that lead to a poor preparation.

\section{BASELINE MEASUREMENT}

The first goal of our project was to determine approximately how many inpatient colonoscopy preparations were rated as poor during a specific period of time. We selected November 2016 to January 2017 as our baseline sample. Colonoscopy reports were reviewed from this period, and the quality of each preparation was recorded. The endoscopists conducting the procedure were the ones originally rating the colonoscopy preparation. These preparation ratings were also then re-confirmed by a third party. Ninety-six preparations from November 2016 to January 2017 were reviewed. In November 2016, 6 out of 25 or $24 \%$ of colonoscopy preparations were rated as poor/inadequate; in December 2016, 5 out of 30 or $17 \%$ were rated as poor/inadequate; and finally in January 7 out of 41 or $17 \%$ were rated as poor or inadequate. This was an overall average of $19 \%$ poor/inadequate rate from November 2016 to January 2017. The goal of the project going forth was to decrease this poor/ inadequate rate. After our interventions were made, the percentage of poor colonoscopy preparations would then be determined each month until July 2017.

\section{DESIGN}

The next step of the project was to determine the factors that contribute to the poor/inadequate rate at our institution. In order to do this, we spoke to multiple people involved in the preparation process. This included nursing staff, clinical aides, residents, fellows, attendings and administrators. We attempted to determine what factors at each of their respective levels were causing poor and inadequate preparations. Their answers were enlightening, as they indicated factors that could be improved on at various levels.

In designing our study, four main categories were evaluated for possible interventions: process, people, materials and environment. These categories are depicted in figure 1.

In terms of process: the colonoscopy preparation process is time intensive for both staff and patients. It requires the patient to drink an unsavoury substance over a period of hours. Checking to see if the preparation worked requires nursing staff to examine the stool for its colour. For physicians, ordering a colonoscopy preparation at our institution was not a simple task. At our institution, there was no standardised, synchronised way to order a full colonoscopy preparation. Orders for the liquid preparation could be placed, but this only included an individual medication order, and did not give instructions about what to do if the preparation could not be completed or was delayed. These types of instructions could be included in an electronic order set, and this was something that was then unavailable for a colonoscopy preparation.

In terms of people: patients usually did not understand how important a good preparation was for their colonoscopy. They knew they had to drink a poor tasting liquid for hours, but did not know why. In addition, the number of staff required in order to administer an adequate preparation was a factor in whether the preparation was successful. If nursing staff was short-handed, they were not able to check the patient as frequently in order to determine the adequacy of the preparation. Physician and nursing participation in the preparation process was also a factor. Physicians and nursing staff often themselves did not understand why a preparation was important. If the importance of a preparation was not known, then the willingness to see the preparation process completed correctly was often not there. Physicians would frequently order the preparation without the needed follow-up to determine whether the preparation was fully taken and adequate.

In terms of materials: the poor taste of the preparation was a factor. Patients did not like to drink the preparation, as it did not taste good. This made it difficult to take and subsequently harder to administrate. In addition, the volume of the preparation was a factor. Patients usually have to drink around $4 \mathrm{~L}$ of preparation in order for it to be successful. This is a large amount, and contributed to the poor preparation.

In terms of environment: the patients who were drinking the preparation were often times very ill. This obviously could impact the administration of the preparation in various ways. In addition, the hospital being busy is a factor, as this can take up time of nursing staff and physicians. Shared patient rooms also contributed to patient's reluctance to fully complete their preparation.

All of these factors were considered in implementing our quality improvement project. Factors that could be addressed easily and directly were identified, and it was 
these factors that we focused on for our project. Specifically, we aimed to focus on three things: patient education on the preparation process; education to physician and nursing staff regarding the preparation process; and the creation of a standardised electronic order set in the electronic medical record (EMR) that would both administer the preparation and give troubleshooting instructions and orders.

\section{STRATEGY}

Once it was determined what factors to intervene on, PDSA cycles were designed for the implementation phase. The three interventions would be completed at the beginning of each month, and the results would be analysed monthly.

\section{PDSA CYCLE 1 (FEBRUARY-MARCH 2017)}

The first PDSA cycle involved education to physicians and nursing staff at J.W. Ruby Memorial Hospital. There are multiple groups of physicians that provide care to the patients at our hospital. In the inpatient setting, the providers that order most of the colonoscopies on our patients are two groups: the medical residents and the hospitalists. The first intervention involved education of these two groups, as well as nursing staff who are also intricately involved in the care of our patients. Education of these groups involved both speaking to them individually and the distribution of a handout. The specifics of the preparation process were explained to these groups. In addition, we explained to the providers and staff various troubleshooting steps that could be undertaken if a preparation was not being successfully completed.

We emphasised a few points about the preparation process:

1. Clear liquids are required during the day prior to the procedure. This allows patients to have clear bowel movements

2. Start the preparation earlier in the evening, around 17:00-18:00, as this gives the patient adequate time to take the preparation successfully

3. The volume and rate of intake are important

4. Follow-up is required

5. When the preparation is not going well, early intervention is required.

We explained various troubleshooting steps to these groups. These included the following:

1. Slow down the preparation intake if needed

2. Anti-emetics/prokinetics can be used in patients who are having difficulty taking the preparation

3. An nasogastric (NG) tube can ultimately be placed in patients who need a preparation, but are unable to complete it on their own

4. If the preparation needs more time to be taken, if needed, the procedure can be delayed if needed

5. As a last resort, magnesium citrate or enemas can be given.
By giving these tips and troubleshooting steps, it was hoped that a decrease in the poor quality rate could be made. The overall percentage of poor inpatient colonoscopy preparations for that month was $11 \%$.

\section{PDSA CYCLE 2 (MARCH -APRIL 2017)}

The second intervention and PDSA cycle was the creation of a standardised order set within our institution's EMR. This order set was comprehensive in that included every order needed to order a colonoscopy preparation. This includes the type of prep; a clear liquid diet the day prior to the procedure; nil by mouth (NPO) orders for the day of the procedure; and various troubleshooting instructions and as needs (PRN) orders. This order set allowed providers to order all the necessary medications and instructions needed for a successful preparation. Specifically, our order set included four different options for diet: NPO now, NPO after midnight, NPO at a certain specified time and a clear liquid diet with no reds or purple liquids. In terms of preparations, two preps were included: a GoLytely prep and a Miralax prep. Under each preparation, we included the option to request a private room for patients; the actual prep order (Miralax or GoLytely); instructions to nursing on when to start the prep and how exactly to administer it; and what do if the patient's stool was not yellow-clear after completing the prep. We also included PRN tap water enemas, and anti-nausea PRNs. There were also orders for nursing staff to contact the primary team if there were any issues with administering the preparation. It was hoped that this order set would streamline and standardise the process for ordering a colonoscopy preparation, as well as increasing communication between the patient, nursing staff and the physicians ordering the preparation. In order to disseminate usage of this order set, an email was sent out hospital wide, alerting attendings, residents and other staff that the order set was now available to be ordered. The overall percentage of poor inpatient colonoscopy preparations for this month was $17 \%$.

\section{PDSA CYCLE 3 (APRIL-MIAY 2017)}

The third intervention and PDSA cycle involved direct patient education. This was a very important intervention, as the patient is the primary person who is required to conduct a successful preparation. We recognised that most patients did not understand why it was important to drink all of their preparation. This partly resulted from a lack of a familiarity with what a successful colonoscopy required. In order to address this, we created a patient handout that explained why a successful bowel preparation was important. We explained to patients that taking a preparation allows their bowels to be adequately emptied. Doing this would allow gastroenterologists to adequately visualise the colon. To depict this, we depicted a picture of a clean, well-prepped bowel versus a poorly prepped bowel. This was the simplest and most effective way of explaining to patients why exactly we needed an 
adequate preparation. We emphasised to the patients that if their colon was unable to be adequately visualised, their procedure may be delayed or cancelled. We explained to them that completing a successful preparation required following their nurse's instructions, and letting them know if there are any issues taking the prep. We ultimately explained to the patient that the end result is a clear, yellow stool that indicates the preparation was successful. It was hoped that by conducting extensive patient education, a meaningful decrease in the rate of poor inpatient colonoscopy preparation could be made. The overall percentage of poor inpatient colonoscopy preparations for this month was $12 \%$.

\section{PDSA CYCLE 4 (MAY-JULY 2017)}

The fourth and final PDSA cycle involved continuing what we determined was likely the strongest intervention, the inpatient colonoscopy order set. During this cycle, the order set was the only intervention that was continued. During this phase, usage of the order set was more widespread as we had already sent an email recommending its use to hospital attendings, residents and staff, as well as recommending its use when giving consult recommendations for inpatient colonoscopies. It was hoped that the widespread usage of the colonoscopy order set would result in a more significant, and long-lasting decrease in the poor preparation rate. The overall percentage of poor inpatient colonoscopy preparations for May was 3\%, June was $5 \%$ and July was 3\%, for a 3-month average of $4 \%$.

\section{RESULTS}

Over a 3-month period, each of the interventions was implemented: direct education on 1 February; order set on $1 \mathrm{March}$; and patient handouts on 1 April. The results were evaluated at the end each month to determine what the effects were. During the initial intervention period (February-April), the results were evaluated each month. Inpatient colonoscopies were evaluated for preparation quality. In the month of February, after the direct education to physicians and nursing staff (PDSA cycle 1), the percentage of poor quality inpatient colonoscopy preparations was $11 \%$ (3 out of 28 preparations). The colonoscopy order set was implemented on 1 March (PDSA cycle 2); for that month, the percentage of poor quality inpatient colonoscopy preparations was $17 \%$ (6 out of 35 preparations). Finally, on 1 April, patient education in the form of patient handouts were implemented (PDSA cycle 3 ); the percentage of poor quality inpatient colonoscopy preparations for that month was 12\% (5 out of 43 preparations). The 3 -month average was thus $13 \%$ poor (14 out of 106 preparations).

As a result of these interventions, over a 3-month period from February to April 2017, the cumulative effect was a decrease in the overall poor preparation rate from $19 \%$ down to $13 \%$ (seen in figures 2 and 3). Education to patients, nursing staff and physicians was a key component in decreasing the rate of poor inpatient colonoscopy

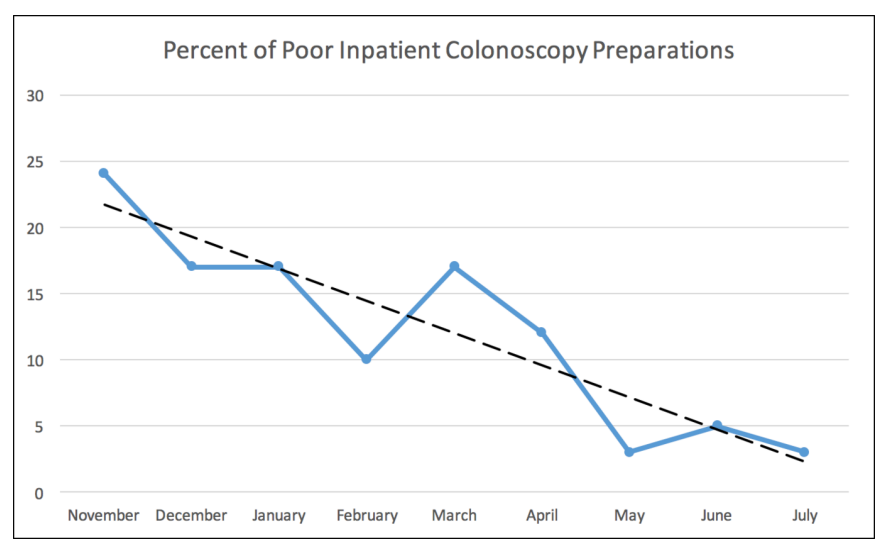

Figure 2 A graph showing a decrease in the overall rate of poor inpatient colonoscopy preparations over a 9-month period.

preparations, as the rate decreased to $11 \%$ in February and $12 \%$ in April, respectively. However, based on the data from February to April 2017, it was then hypothesised that the inpatient colonoscopy order set was the intervention that resulted in the greatest impact, even though it was not initially reflected in the monthly data. The month that the order set was initially implemented (March) resulted in a poor inpatient colonoscopy preparation rate of $17 \%$, which was higher compared with the other two interventions. We believed this was an outlier. The reasons for this higher rate were likely due to the time it took for the use of the order set to become widespread. An email was sent hospital wide that the order set was now available, and it was believed that this led to its increased usage throughout the hospital. In order to confirm our hypothesis and determine the long-term effects of the order set, this was the only intervention that we continued during the follow-up months of May, June and July (PDSA cycle 4). It was hypothesised that as the order set gained popularity in the hospital, its positive effects would continue and lead to further decreases in the rate of poor inpatient colonoscopy preparations.

This hypothesis was confirmed. We continued to monitor and evaluate the quality of the inpatient colonoscopy preparations throughout May, June and July. The results for this period indicated that the order set was indeed the strongest intervention. For the month of May, the percentage of inpatient colonoscopies preparations rated as poor was $3 \%$ (1 out of 33 preparations). For the month of June, the percentage of inpatient colonoscopies

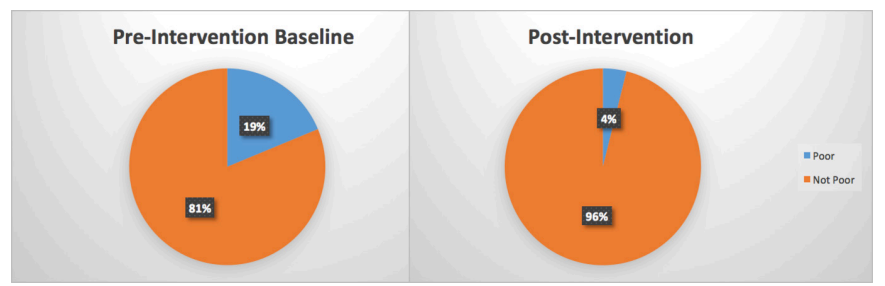

Figure 3 Pre-intervention and postintervention measurements showing the decrease in the percentage of poor preparations versus not poor over a 3-month period. 
preparations rated as poor was $5 \%$ (2 out of 37 preparations). For the month of July, the percentage of inpatient colonoscopies preparations rated as poor was 3\% (1 out of 33 preparations). Thus, the overall rate of poor inpatient colonoscopy preparations from May to July 2017 was $4 \%$, which is a significant decrease from the initial baseline of 19\% from November to January 2016.

\section{LESSONS AND LIMITATIONS}

Many lessons were learnt during this quality improvement project. First, the participation and input of many different staff members was required in order conduct this project. The factors affecting poor inpatient colonoscopy preparations were complex, and distributed across different levels of care. At our institution, input was received from nursing staff, hospitalists, residents and other staff members. Each of them brought their own perspective about what contributed to a poor colonoscopy preparation. One of the biggest lessons learnt was that communication, or a lack thereof, is one of biggest impediments to a successful preparation. This can be demonstrated in multiple ways: a lack of communicating to the patient why the preparation is important in the first place; a lack of communicating to nursing staff-specific preparation instructions; and a lack of communication between nursing staff and physicians. Communication is key in ordering a preparation, and we thought that our project intervened on increasing communication among multiple groups in the hospital. Another lesson we learnt was that implementing a system wide change, and seeing its results, will take time. We believe that the implementation of the order set was the strongest intervention we made; however, our initial data showed an increase in the poor colonoscopy preparation rate. We learnt that as time went on and knowledge of the availability of the order set grew, the overall poor preparation rate eventually decreased significantly. Thus, patience is needed when implementing a change to the previous system.

There are various limitations to consider in our study. First, the preparations in our studies were rated by the endoscopists themselves; in order to reduce observer bias, these ratings were again checked by a third party. There may be limitations in that there is a certain degree of interobserver variability among different physicians in rating the preparations. This could be reduced in future studies by using standardised scales such as the Boston Bowel Preparation Scale. ${ }^{3}$ However, even with the use of the Boston Scale and other scales such as the Chicago Bowel Preparation Scale, there is an element of subjectivity that cannot be removed, and thus complete variability cannot be eliminated. ${ }^{4}$ Another possible limitation to our study is generalisability. Our quality improvement project was conducted at a single medical centre, and thus each institution may have its own policies and procedures for administering preparations. However, we do believe that the general interventions that we have outlined above can be implemented at other institutions. Education was a key step in our project that can be applied elsewhere. Explaining the process and importance of a successful colonoscopy preparation to patients, hospitalists, residents, nursing staff and others can result in a decrease in the rate of poor inpatient colonoscopy preparations. Furthermore, for the greatest impact, we believe that institutions that use an EMR should build ways to standardise and simplify the process of ordering a colonoscopy preparation. At our institution, this resulted in the creation of an electronic order set. A successful order set should also include troubleshooting steps for nursing staff, as well as instructions on how and when to communicate the ongoing results of the preparation. Combined, these steps can result in a significant decrease in the rate of poor inpatient colonoscopy preparations.

In terms of sustainability, we felt that the creation of the order set was the most sustainable intervention, as it is something that is now permanently within the electronic medical system. Educational interventions were less sustainable, as these depended on individual behaviour and retention; however, we do think that in the short term, educational interventions are also helpful, and do help contribute to better colonoscopy preparations. In this regard, at our institution, we will continue to have annual meetings with physicians and nursing staff, as well as distribute patient education handouts. One additional limitation to this study is that in regard to the educational interventions, learner knowledge was not tested pre-intervention and postintervention. However, we do feel that the noted decreases in the poor colonoscopy rate during the months of educational intervention were a measure of a successful change, attributable to the educational interventions. Future studies could look more in depth at the before and after effects of educational intervention on learner knowledge and colonoscopy preparations.

\section{CONCLUSION}

The cost of a colonoscopy can be anywhere from around several hundred to several thousand dollars. Poor colonoscopy preparations can lead to repeat procedures that can be a significant cost to both the patient and the healthcare system. This can in turn lead to longer length of stays, less patient satisfaction and worse outcomes. The results of our study show that the PDSA approach can be used successfully in the setting of inpatient colonoscopy preparations. By using our three interventions (physician/nursing education, patient education and the creation of an order set), we were able to achieve a decrease in the rate of poor inpatient colonoscopy preparations from a 3-month average of $19 \%$ down to $4 \%$. More specifically, we believe the use of an electronic order set to order inpatient colonoscopy preparations resulted in the greatest impact. We hope that the interventions we have conducted can be replicated and applied to other institutions, and thus result in 
better outcomes for patients, providers and healthcare facilities.

Contributors All three authors (SMS-K, JC and GJR) were involved in the design, implementation, analysis and interpretation of the work. All three authors were involved in the drafting and revising of the work. All three authors approved the final version to be published. All three agree to be accountable for all aspects of the work, and all three are guarantors of the work. SMS-K is the corresponding author. Competing interests None declared.

Provenance and peer review Not commissioned; externally peer reviewed.

Open Access This is an Open Access article distributed in accordance with the Creative Commons Attribution Non Commercial (CC BY-NC 4.0) license, which permits others to distribute, remix, adapt, build upon this work non-commercially, and license their derivative works on different terms, provided the original work is properly cited and the use is non-commercial. See: http://creativecommons.org/ licenses/by-nc/4.0/
(C) Published by the BMJ Publishing Group Limited. For permission to use (where not already granted under a licence) please go to http://www.bmj.com/company/ products-services/rights-and-licensing/

\section{REFERENCES}

1. Brahmania M, Park J, Svarta S, et al. Incomplete colonoscopy: maximizing completion rates of gastroenterologists. Can J Gastroenterol 2012;26:589-92.

2. Calderwood AH, Mahoney EM, Jacobson BC. A plan-do-study-act approach to improving bowel preparation quality. Am J Med Qual 2017;32:194-200.

3. Gerard DP, Foster DB, Raiser MW, et al. Validation of a new bowel preparation scale for measuring colon cleansing for colonoscopy: the chicago bowel preparation scale. Clin Trans/ Gastroenterol 2013;4:e43.

4. Lai EJ, Calderwood AH, Doros G, et al. The Boston bowel preparation scale: a valid and reliable instrument for colonoscopy-oriented research. Gastrointest Endosc 2009;69:620-5. 